\title{
Reducing environmental pollution by chemical herbicides using natural plant derivatives - allelopathy effect
}

\author{
Ghassan Faris Al-Samarai ${ }^{1, A-F}$, Wael.M. Mahdi ${ }^{1, A-C}$, Baraa Mohammed Al-Hilali ${ }^{1, D-F}$ \\ ${ }^{1}$ Department of Biology, College of Education, University of Samarra, Samarra, Iraq \\ A - Research concept and design, B - Collection and/or assembly of data, C - Data analysis and interpretation, \\ $D$ - Writing the article, E - Critical revision of the article, F- Final approval of article
}

Ghassan Faris Al-Samarai, Wael.M. Mahdi, Baraa M Hilali. Reducing environmental pollution by chemical herbicides using natural plant derivatives - allelopathy effect. Ann Agric Environ Med. 2018; 25(3): 449-452. doi: 10.26444/aaem/90888

\begin{abstract}
Introduction. Weeds are an important factor in determining production in the agricultural sector. Currently, weeds have been managed by employing synthetic weedicides chemicals. These chemicals have a negative impact on the environment through accumulation in soil and water, in addition to their effect on biological diversity.

Materials and method. A study was undertaken to evaluate the activity of the aqueous extract of leaves from; Nerium (Nerium oleander L), olives (Olea europaea L) and castor (Ricinus communis L) on the control of Cyperus rotundus L. The study comprised of two components: three plant species, and the uses of three planta extracts concentration (5\%, $7.5 \%$ and $10 \%)$ and their impact on germination and inhibition of seedling growth.

Results. The results from using Nerium extract in comparison with Tibenuron and the two other plants extracts gave $90 \%$ inhibition at concentrations of $5 \%$ and $10 \%$, while the concentration of $2.5 \%$ gave the lowest inhibition rates for germination of all plant extracts under study. The second experiment carried out using $10 \%$ concentration of the Nerium extract gave complete inhibition (100\%), compared with the same concentrations of other plants extract and weedicides.

Conclusions. The current study indicates the possibility of using natural products of plant origin as alternatives to chemical herbicides as one of the means of biological control to reducing environment pollution.
\end{abstract}

\section{Key words}

chemical herbicides, environmental pollution, allelopathy, plant derivatives

\section{INTRODUCTION}

Weeds are an important factor influencing crop production through competition for environmental resources and cause heavy production losses [1]. According to reports of Nishimoto [2] weeds caused damage reached $14 \%$ of total agricultural production. Cyperus is one of the most prevalent weeds worldwide, is more resistant to traditional control methods because of its ability to survive under critical environmental conditions, and for rapid reproduction through base tubers [3]. Through the increasing demand for food production, particularly agricultural crops, there is an urgent need to find ways and means to control weeds, since weeds compete with crops for light, water and nutrient in the soils. The reduction of their proliferation gave remarkable results in accord with the growing demand for fresh agricultural products to meet the needs of the market and consumers $[4,5]$. Demand for the use of manufactured plant chemical herbicides has grown and has become one of the most important ways to combat weeds Cybros [6].

There is increasing global interest in reducing the use of synthetis herbicides in the agricultural sector because of the continued human exposure to the minimum levels of herbicides and accumulation through their diet $[7,8$, 9]. In fact, chemical herbicides exceed even the maximum allowable residue that allows for the accumulation in fresh

Address for correspondence: Ghassan Faris Al-Samarai, University of Samarra, 34010 Samarra, Iraq

e-mail: ghfaat76@gmail.com

Received: 12.01.2018; accepted: 09.05.2018; first published: 17.07.2018 fruits in accordance with international standards. In addition to the negative impact of herbicides on human health and the environment, there is the emergence of new strains resistant to chemical herbicides $[10,11]$. Nevertheless, excessive use of weed herbicides has had led to the emergence of new strains resistant to chemical herbicides. According to the Nishimoto report [2], many types of chemical-resistant weeds have been recorded. The above scenario led to the search for a safe alternatives to artificial herbicides to control weeds in the agricultural sector and minimize losses, and avoid the negative effects of the use of synthetics weedicides. There are a few research works investigating and testing naturalproducts for the capability to stop the germination of the seeds of the weeds (herbicides). Due to increasing emphasis on organic agriculture and environmental protection, attention has been paid to Allelopathy research. The physiological and ecological mechanisms of Allelopathy are gradually being elucidated and it is obvious that Allelopathy requires further research and widespread application in agricultural production worldwide [12] Current research focuses on the use of a biological control method using plant extracts as a serious and beneficial alternative to chemical weedicides in controlling weeds. The aim of this study is to investigate the use of plant extracts as one of the proposed solutions (Allelopathy) for the use of a natural and compatible method as an effective alternative in the management of weed (Cyperus rotundus $\mathrm{L}$ ). 


\section{MATERIALS AND METHOD}

Selection and collection of plant samples. Tubers were collected from the village of Al-Abbasiya/Samarra district of the agricultural lands located on the banks of the Tigris River, where the grass grows densely in fields planted with crops. The tubers wre healthy, large in size and free of any pathological condition. The plants were collected, and brought to the laboratory, and washed in tap water to remove dust. The tubers were left for one day, until dry, and then kept in a plastic container until use.

Three plants that are grown and spread over almost the whole of Iraq (Tab. 1) were selected for the preparation of plant extracts, which were tested for their ability to inhibit the germination of weed seeds

Table 1. Types of plant under study

\begin{tabular}{cc}
\hline Scientific name & Common name \\
\hline Nerium oleander. L & Nerium \\
\hline Olea europaea. $\mathrm{L}$ & Olive \\
\hline Ricinus communis. L & Castor \\
\hline
\end{tabular}

After selecting the plants, fresh leaves collected directly from the trees growing on the outskirts of the city of Samarra were brought to the laboratory and washed carefully in tap water to remove dust and dirt, and then washed with distilled water. The plants left in the laboratory were continuously stirred daily to avoid rot until they were completely dry. The samples were placed in perforated paper bags and then placed in an oven at $45^{\circ} \mathrm{C}$ for 48 hours for complete dehydration. After that, the samples were crushed, using a sieve to exclude the non-grinding hard parts, followed by use of a blender to obtain dry powder. Samples of plant powders were kept in clean, sealed and placed in sealed glass containers for easy preservation and storage [13].

Preparation of extracts. The stored powder prepared previously was extract by water [14]. The powders were immersed in water with continuous stirring until the homogenization state was reached, and then left for 48 hours at $4^{\circ} \mathrm{C}$ in a refrigerator. The solution was then filtered in two stages, the first stage by using a soft cloth to remove large impurities, and the second stage using filter paper (Whatman, 2) to obtain a crude extracts solution. The crude extracts mixture was desiccated using a rotary evaporator to finally obtain the crude extraction. After that, a series of dilutions of the crude mixture were prepared $(10 \%, 7.5 \%$ and 5\%) and kept in sterilized plastic containers until use.

Design experiments. The experiments were carried out according to the design of random sectors (CRD) with two factors: 1) 3 types of plant, and 2) 3 concentrations (5\%, $7.5 \%$ and $10 \%$ ). In addition to (2), control plants (water + Tibenuron weedicide) with 4 replicates (Tab. 2).
Table 2. Treatments of study

\begin{tabular}{|c|c|c|c|c|}
\hline $10 \%$ & \multirow{3}{*}{ Olive } & T5 & Control & T0 \\
\hline $7.5 \%$ & & T6 & \multirow{2}{*}{ Herbicides } & \\
\hline $5 \%$ & & T7 & & \\
\hline $10 \%$ & \multirow{3}{*}{ Castor } & T8 & $10 \%$ & $\mathrm{~T} 2$ \\
\hline $7.5 \%$ & & T9 & Nerium & $\mathrm{T} 3$ \\
\hline $5 \%$ & & $\mathrm{~T} 10$ & $5 \%$ & $\mathrm{~T} 4$ \\
\hline
\end{tabular}

\section{Experimental Study}

Germination Rate. Soil which had been prepared for seeding was irrigated until reached to optimum watering and eliminated blanks by using water. Seeds were planted in prepared soil (4 tubers per lentil) and regularly watered (every 2 days) with extracts. The experiment was checked daily and the germination rate recorded for all treatments for a period 2 weeks. Tuber germination rate was calculated according to the method of [15], following the equation 1.

Inhibition of seedling growth. Tubers planted in previously prepared soil were left until the stage of seedling was reached (range between 10-15 cm). After that, the seedling was irrigated with plant extracts every 2 days for one month. The data collected, based on the percentage of damage and killing of the irrigated seedling, utilised the equation 2.

Statistical analysis. Statistical analysis was carried out using statistical software SPSS version 19- (SPSS Inc., Chicago, USA). A variety ANOVA was used to analyse the experimental results of dependent variables. Significant differences between mean values were determined using Duncan's Multiple Range test (DMRT) $(\mathrm{P} \leq 0.05)$. All data presented were expressed as mean \pm standard error.

\section{RESULTS AND DISCUSSION}

Influence of plant species crude extracts and concentrations on germination. The percentage of inhibition of tubers germination of Cyperus rotundus L. irrigated with leaf crude extracts of; Nerium, Castor and Olive compared with herbicide is shown in Figure 1. The results showed significant differences between the treatments and controls (water only, chemical weedicide). The highest percentage of inhibition of the germination was recorded from soil irrigated by crude extract obtained from Nerium. The percentage of inhibition reached $80 \%$, followed by castor leaf extract (35\%), while olive plant extract produced the lowest rate of inhibition (23\%).

Figure 2 shows the effect of using different concentrations of the aqueous extract under study on the germination of irrigated tubers. There was a significant effect of the concentrations $(10 \%$ and $7.5 \%)$ of Nerium extract on the rate of inhibition of tubers with values approximately reached $90 \%$ for both concentrations, compared with other treatments. Concentrations of $10 \%, 7.5 \%$ and $5 \%$ from olive leaf extract

Rate of germination rate $=\frac{\text { control plants }- \text { percentage of tubers germination (irrigated with extracts) }}{\text { Tubing germination ratio for control }} \times 100 \quad$ Equ. 1

Percentage of inhibition $=\frac{\text { No. Plants in control treatment }- \text { No. treated plants (irrigated with extracts) }}{\text { Number of plants in control treatment }} \times 100 \quad$ Equ. 2 


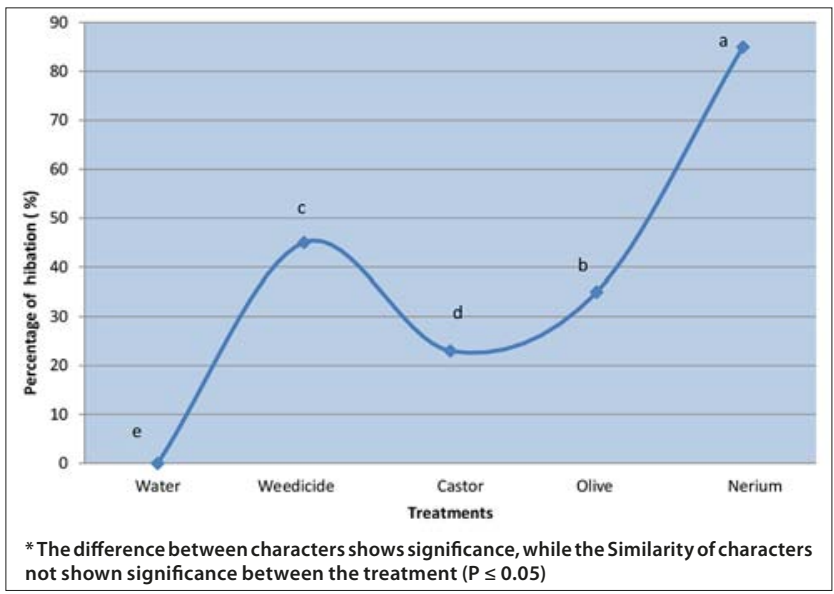

Figure 1. Percentage of inhibition germination tubers (\%) of Cyperus rotundus L. irrigated with leave crude extract of (Nerium, Castor and Olive) compared with herbicide

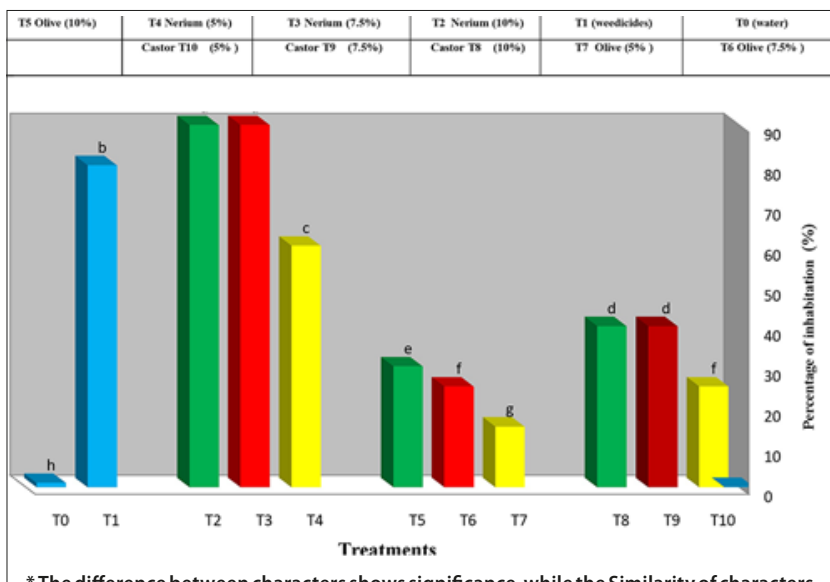

* The difference between characters shows significance, while the Similarity of characters not shown significance between the treatment $(P \leq 0.05)$

Figure 2. Percentage of inhabitation germination tubers (\%) of Cyperus rotundus L. using different concentrations (10, 7.5 and $5 \%$ ) from Nerium, Olive and Castor extract compared with herbicide

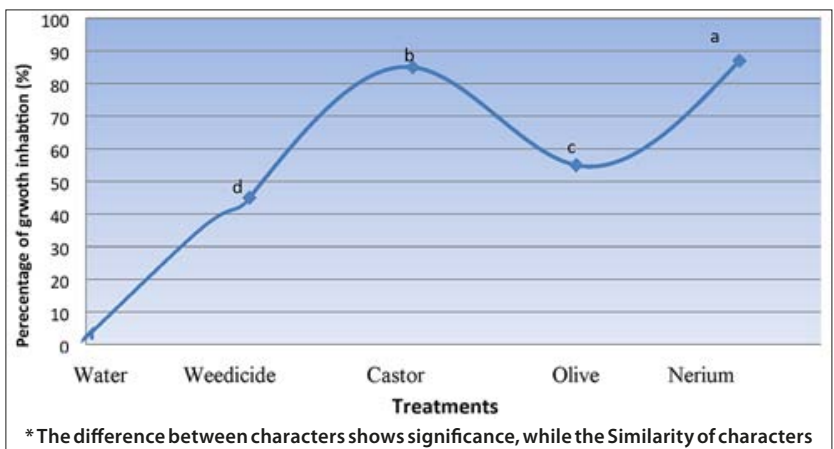

not shown significance between the treatment $(P \leq 0.05)$

Figure 3. Percentage of growth inhibition seeding (\%) of Cyperus rotundus $L$. irrigated with leave crude extract of (Nerium, Castor and Olive) compared with herbicide

gave the lowest inhibition percentage values - 25\%, 25\% and $15 \%$, respectively. On the other hand, it was noted that an increase in the inhibition ratios were in accordance with the rise in dose concentration for all studied plants.

From the above result, the extract of Nerium showed a higher inhibitory effect on tubers germination than that of Olive and Castor. The effect of inhibition in germination can be attributed to the congenital effects of plant containment in soluble substances in water. On the other hand, the difference in inactivation of tuber germination by different plant species

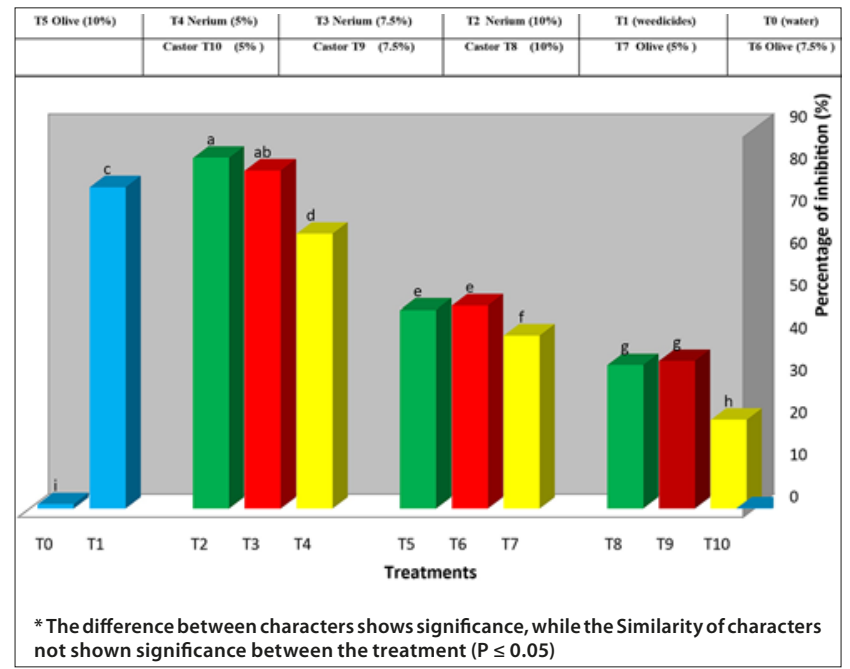

Figure 4. Percentage of growth inhibition seeding (\%) of Cyperus rotundus L. using different concetrations (10, 7.5 and 5\%) from Nerium, Olive and Castor extract compared with herbicide

may be attributed to differences in the chemical composition of these plants. The inhibitory effect of Allelopathy is complex and can involve interaction between different classes of chemicals, such as: phenolic compounds, flavonoids, terpenoids, alkaloids, steroids, carbohydrates, amino acids or a mixture of different chemical compounds that sometimes have a toxic effect greater than individual compounds [16]. The results of the presented study coincide with the results of [17], in that the Rhyme extract inhibited the germination of Egyptian Parsia and Benicetom divisum seeds. A study carried out by [18] listed plant extract components which may include a wide range of inhibitory ingredients through their effect on alkaloids photosynthesis, mitochondrial function, plant hormones, ion absorption and water balance. The inhibitory activity of the Nerium plant may be due to glucosinolate, which accumulates in large quantities in the plant parts, especially the leaves. Although it is toxic to human and animals, it has also proved to contain medicinal components, such as antibacterial and anti-inflammatory activity [21].

Influence of plant type and concentration on the growth of the seedling. The influence of plant species and concentration on the growth of the seedling is presented in Figure 4. Seedlings growing to around $10-15 \mathrm{~cm}$ were irrigated with plant extracts at concentrations of 10, 7.5 and 5\%). The effect was evaluated on the basis of the rate of inhibition of seedling. The crude extracts of Nerium and Castor gave the highest growth inhibition effect on seedling with values of $87 \%$ and $85.5 \%$, respectively. These values exceeded the effect of the Olive extract in comparison with control. The lowest rate of growth inhibition was recorded from seedling irrigated with Olive extract.The average reached approximately 55\% (Fig. 3).

The effect of different extract concentrations $(10 \%, 7.5 \%$, and 5\%) from plants under study on the growth of weed seedling is shown in Figure 4. The highest inhibition rate was recorded using the concentration of $10 \%$ and $7.5 \%$ from the Nerium plant with values of $82 \%$ and $77 \%$, espectively. At the same time, thee concentrations produced significant differences from all the other concentrations of the same plant - Nerium), and Olive and Castor plants. It was observed that treatment of the control using chemical weedicide recorded high levels of growth inhibiting of seedling of Cyperus, with 
a value of $74 \%$ compared with Castor and Olive plant extracts and at all concentrations. Olive plant extract recorded the lowest percentage in inhibiting growth seedling with values of $20 \%, 32 \%$ and $34 \%$ for concentrations of $5,7.5$ and $10 \%$, respectively.

It was observed that the impact of plant extracts ranged between strong and weak regarding germination and seedling growth. This result is in agreement with the findings of Olofsdotter et. al. [22] who found that the aqueous extracts of the annual weeds had variation between them in the effects on the germination, which ranged from negative to positive activity. The findings of other researchers $[23,24,25,26,27]$ are consistent with the current results. They too reported a high rate of inhibition of weed growth by employing various plant extracts. The results were in agreement with the findings of $[28,29]$, in that the water extract of many plant extracts showed a positive effect on the inhibition of seed germination, seedling length, and dry leg weight of all weeds studied. Based on the presented research results, there are possibilities to use the plant species used in this study from Iraqi flora as an alternative to using weedicides to control Cyperus rotundus $\mathrm{L}$.

\section{CONCLUSION}

The crude extract of the plants Nerium and Castor produced a positive effect on inhibiting tubers and seedling growth; hence, the possibility of using them in eco-friendly, biocontrol programme management. In conclusion, more future studies are required to support the results of this study regarding the control of Cyperus rotundus $\mathrm{L}$.

\section{Acknowledgements}

The authors wish to thank the staff and colleagues in the Department of Biology at the College of Education of the University of Samarra, Iraq, for providing facilities to complete this research.

\section{REFERENCES}

1. Hossain M, Begum M. Soil weed seed bank: Importance and management for sustainable crop production- A Review Bangladesh Agriculture Univ. 2015; 13(2): 221-228.

2. Food and Agriculture Organization.FAO. Weed management for developing countries.2011; (Addendum 1).

3. Nishimoto R. Purple nutsedge tuber sprouting. Preserve Commission, Latham, New York. 2001. 50 pps.

4. Rana Al-Dulaimi. Norli I.Mahamad H. Responses of growth of lady's fingers (Abelmoschus esculentus L.) to different treatments methods of dairy wastewater. Ann Agric Environ Med. 2014; 21(1): 42-48.

5. Hawver CA, Gifford SB, Hecht J. Comparison of methods to controlinvasive plant species in the Albany Pine Bush Preserve.2000; Albany Pine Bush.

6. Med WA, Dwaipayan S, Ashim C. Impact of herbicides use in agriculture: their benefits and hazards. Interdiscip Toxicol. 2009; 2(1): 1-12.
7. Japan Food Chemical Research Foundation JFCRF. Maximum Residue Limits (MRLs) of Agricultural Chemicals in Foods; 2011.

8. Food and Agriculture Organization.FAO.Implications of Maximum Residue Levels (MRLs) on tea trade; 2011.

9. Al-Samarrai G, Harbant S, Muhammad S. Evaluating eco-friendly botanicals (natural plant extracts) as alternatives to synthetic fungicides. Ann Agric Environ Med. 2012; 19(4): 673-676.

10. Vladimir B, Florence Y, Li F. History of arsenical herbicides and health risks related to the use of Agent Blue. Ann Agric Environ Med. 2017; 24(2): 312-316.

11. Cheng F, Zhihui F. Research Progress on the use of Plant Allelopathy in Agriculture and the Physiological and Ecological Mechanisms of Allelopathy. Frontiers Plant Sci. 2015; (6): 1020. doi:10.3389/ fpls.2015.01020.

12. Ruch B. Processing of neem for plant protection simple and sophisticated standardized extracts. Abstracts of the.Work shop, Neem and Pheromones, University of Uberaba, Brazil.2001; March 29-30 Augusts, P.499.

13. Francisco DH, Francisco CG, Raul R. Lippia graveolens and Carya illinoensis Organic Extracts and there in vitro Effect against Rhizoctonia Solani Kuhn. Am J Agric Biol Sci. 2012; 5(3): 380-384.

14. Singh G, Bhati M. Mineral Accumulation, Growth, and Physiological functions in Dalbergia sissoo Seedlings Irrigated with Different Wastewaters. J. Environ. Sci. Health Part A-Toxic/Hazardous Substances \& Environmental Engineering. 2003: (38): 2679-2695.

15. Bangarwa SK, NorsworthyJK, Grub EE. Effect of Turnip Soil Amendment and Yellow Nutsedge (Cyperus esculentus) Tuber Densities on Interference in Polyethylene-Mulched Tomato. Weed Technology. 2012; (26): 364-370.

16. Saeed AM, Al-Dos AA. Effect of allopathic plant Rhizya stricta on germination with some plants Pasture. Annu Agric J. 1997; 42 (1): 159-167.

17. Majeed A, Chaudhry Z, Muhammad Z. Allelopathic assessment of fresh aqueous extracts of Chenopodium album L. for growth and yield of wheat (Triticum aestivum L.). Pak J Bot. 2012; 44(1): 165-167.

18. Zimer E. Nerium oleander Linnaeus. 2009: 1753.

19. Derwich E, Benzian Z, Boukir A. Antibacterial Activity and Chemical Composition of The Essential Oil from Flowers of Nerium oleander. Electronic J Environ Agric Food Chem. 2010; 9(6): 1074-1084.

20. Kirbag S, Zengin F, Kursat M. Antimicrobial Activities of Extracts of some Plants. Pakistan J Botany 2009; 41(4): 2067-2070.

21. Reigosa MJ, Gonzales L, Souto XC, Pastoriza JE. Allelopathy in forest ecosystems Kluwer Acadmic publishaers. 2000; 184-193

22. Duke SO, Dayan FE, Rimando AM. Natural products as tools for weed management. Weed Res. 2000; 40(1): 99-111.

23. Olofsdotter M, Jensen LB, Courtois B. Improving crop competitive ability using allelopathy - an example from rice. Plant Breeding 2012; 121(1): 1-9.

24. El-Rokiek KG, El-Shahawy TA, Sharara FA. New approach to use rice straw waste for weed control II. The effect of rice straw extract and fusilade (herbicide) on some weeds infesting soybean (Glycan max L). Int J Agric Biol. 2006; 8(2): 269-275.

25. El-Rokiek KG, Aid RA. Allelopathic effects of Eucalyptus citriodora o amaryllis and associated grassy weed. Planta Daninha. 2009; 27: 887-899.

26. El-Rokiek KG, El-Din SAS, Sharara FAA. Allelopathic behaviour of Cyperus rotundus L. on both Chorchorus olitorius (broad leaved weed) and Echinochloa crus-galli (grassy weed) associated with soybean. J Plant Prot Res. 2010; 50(3): 274-279.

27. Jabran K. Wheat Allelopathy for Weed Control. In: Manipulation of Allelopathic Crops for Weed Control. SpringerBriefs in Plant Science. 2017. Springer, Cham

28. Jabran K, Farooq M. Implications of potential allelopathic crops in agricultural systems. In Allelopathy.2013; (pp. 349-385). Berlin: Springer.

29. Joanna J, Wojciech H, Wojciech S, Danuta L. Exposure to phenoxyacetic acid herbicides and predictors of exposure among spouses of farmers. Ann Agric Environ Med. 2012; (19):1.51-56. 